\title{
Red blood cells affect the margination of microparticles in synthetic microcapillaries and intravital microcirculation as a function of their size and shape
}

Rosa D'Apolito ${ }^{1,4}$, Giovanna Tomaiuolo ${ }^{1,4 *}$, Francesca Taraballi $^{2}$, Silvia Minardi $^{2}$, Dickson Kirui ${ }^{2}$, Xuewu Liu $^{2}$, Armando Cevenini ${ }^{3}$, Roberto Palomba ${ }^{2}$, Mauro Ferrari ${ }^{2}$, Francesco Salvatore ${ }^{3,4}$, Ennio Tasciotti ${ }^{2}$ andStefano Guido ${ }^{1,4}$

1 Dipartimento di Ingegneria Chimica, dei Materiali e della Produzione Industriale, Università di Napoli Federico II, Italy

2 Department of NanoMedicine, Houston Methodist Research Institute, Houston, TX, USA

3 Department of Molecular Medicine and Medical Biotechnology, Università di Napoli Federico II, Italy

4 CEINGEBiotecnologie avanzate, Napoli, Italy

5 NavalMedicalResearch Unit, San Antonio, TX

* Corresponding author: Tel.: ++39 081 7682261; Fax: ++39 081 2391800; Email: g.tomaiuolo@unina.it

The first twoauthorscontributedequally to this work.

\begin{abstract}
A key step in particle-based drug delivery through microcirculation is particle migration from blood flow to vessel walls, also known as "margination", which promotes particle contact and adhesion to the vessel wall. Margination and adhesion should be independently addressed as two distinct phenomena, considering that the former is a fundamental prerequisite to achieve particles adhesion and subsequent extravasation. Although margination has been modeled by numerical simulations and investigated in model systems in vitro, experimental studies including red blood cells (RBCs) are lacking. Here, we evaluate the effect of RBCs on margination through microfluidic studies in vitro and by intravital microscopy in vivo. We show that margination, which is almost absent when particles are suspended in a cell-free medium, is drastically enhanced by RBCs. This effect is sizeand shape-dependent, larger spherical/discoid particles being more effectively marginated both in vitro and in vivo. Our findings can be explained by the collision of particles with RBCs that induces the drifting of the particles towards the vessel walls where they become trapped in the cell-free layer. These results are relevant for the design of drug delivery strategies based on systemically administered carriers.
\end{abstract}

Keywords:Margination, Microcirculation, Red blood cells, Drug delivery, micro-particles, Size, Shape.

\section{Introduction}

In recent years, the use of nano- and micro-scale systems as means to deliver drugs has emerged as a powerful tool in the treatment of cancer, pain, infections and inflammatory disorders [1-4]. In fact, drug delivery particles have many advantages, namely, targeted delivery, reduced side-effects, controlled releaseas well as improved pharmacokinetics and a better therapeutic index [4, 5]. Particlesfor drug delivery are usually injected intothe bloodstream at systemic level and, before reaching their final biological target (diseased cells), they must overcomethe barriers present in the body[6]. An essential step in thisjourney - recently defined an odyssey[7] - is particle transport in 
microcirculation, which is where most of the exchangeof nutrients, oxygen, $\mathrm{CO}_{2}$ and other metabolites between blood and tissues occurs. Other key steps of thisjourney are migration towards vessel walls, near wall accumulation (also known as margination[8]), direct contact with the wall,firm adhesion and extravasationfrom the microvasculature to the surrounding tissues[7].Marginationin particular promotesparticle contact and adhesion to the vessel wall, so, although margination and adhesion are two distinct phenomena, the latter is strongly dependent on the former. Based on this concept, adhesion is sometimes considered the hallmark of margination, which is studied by measuring the particles adhered to a surface under flow conditions [9]. However, this approach does not take into account the flow-induced migration and distribution of particles - a factor that must be considered in studies aimed at elucidating the mechanisms governing margination. Furthermore, while marginationdepends mainly on bio-mechanical and geometrical features[10-12], adhesion may be also affected by other factors (i.e., biochemical signaling, receptor/ligand density, and type of target) [13]. Therefore, the two phenomena should be independently addressed, considering that margination is a fundamental prerequisite to achieve particles adhesion and the subsequent extravasation as particles travel to the target site.

In this study we focused on marginationand on the effect exerted by red blood cells (RBCs) on the flow of particles throughmicrocapillaries. The particulatenature of blood[14, 15] and the deformability of RBCs [16-23] are key factors in the transport and distribution of RBCs.This isillustrated by the well-known margination of platelets and white blood cells in human microvasculature [12, 24-27], which can beattributedto the combination of three phenomena: i) the migration of RBCs towards the vessel centerline due to a hydrodynamic lift - a process that leaves a cell-free layer near the vessel wall [23, 28-30]; ii) the cross-flow migration of platelets towards the vessel wall due to their collisions with $\mathrm{RBCs}$, which promotes a lateral displacement[25, 28, 31]; iii)the concentration of platelets in the cell-free layer near the wall, due to their size, shape and rigidity thereby allowing a rigid-body flipping motion near the wall due to the less collision frequency with RBCs. Specifically, marginationof particles consists of two steps: shear-induced diffusion, promoted by RBC-particle collisions that take place in the RBC-rich core, and a fast lateral displacement, called 'waterfall effect', that occurs when particles approach the cell-free layer[28, 31]. The first step is the well-known slow diffusion already described for platelets (step ii) in the previous list)[32]; the waterfall effect, instead, is a result of the inhomogeneity of RBC spatial distribution that generates bypass regions through which particles can rapidly reach the cell-free layer.

In analogy with platelets, drug-carrierswithin the bloodstream are also expected to migrate along the vessel radius [12] and to concentrate in the cell-free layer near the vessel wall.The migration of particles of different sizes and shapesin blood flow models has been the subject of mathematical simulations and experimental research. Some computational studies showed that microparticles(diameter about 1-2 $\mu \mathrm{m}$ )tend to accumulate near the tube wall to a greater extent than nano-particles(diameter about $100 \mathrm{~nm}$ ), which present only a limited near-wall accumulation when flowing in the presence of RBCs [30, 33]. Otherstudies, based on mathematical models[34], reported efficient marginationbothfor spherical versus ellipsoidal particles [35], andfor discoidwith a low aspect ratio versus spheres [31, 36, 37].

Most experimental studies addressedmargination of micro- and nano-particles under fluid dynamic conditions that mimickedthe microcirculation, but in the absence of bloodcellularcomponents [38, 39], thus not taking into consideration the key role played by RBCs, and, moreover, most of the articles on particle margination in blood flowin the presence of RBCsfocused on nanoparticle 
margination[23]. It has recently been reported that micro-particles outperform nano-particles in the propensity to migratetowards the endothelial layer, as reported for spherical [9] and ellipsoidal particles[40]. However, the environmental conditions in the latter studies (i.e., rectangular channels) are far from the ones actually experienced in the human microcirculation, which makes it difficult to evaluate the clinical relevance of the results. From the experimental standpoint, one of the main difficulties is to create a physiologically relevant model of microcirculation in which the flow of individual particles and their interactions with RBCs and vessel walls can be monitored in real time. The development of microfluidicstechniques, based on the fabrication of microchannels and on the accurate control of blood flow, has furthered our understanding of flow dynamics in the human microvasculature, thereby creatingthenew Blood-on-a-chip research field [18, 41]. Microfluidics has variousadvantages, namely, the ability to mimic the network of channels present in the human microvasculature [18] and to apply alaminar flow regime(as found in the microcirculation), which, together with the very small quantities of samples required $[42,43]$, make it the ideal tool with which to study marginationin vitro.

Here, we used a blood-on-a-chipapproach to investigate the effect of RBCs on margination of micro-particles ( $\mu$-Ps)in relation totheir size and shape. In particular, we chose three representativeshapes: spherical $(1 \mu \mathrm{m}$ and $3 \mu \mathrm{m})$,discoidal $(400 \mathrm{~nm} \times 1 \mu \mathrm{m})$ and rod-like $(400 \mathrm{~nm} \mathrm{x}$ $1.8 \mu \mathrm{m}$ ). Micro-particles (up to $1 \mu \mathrm{m}$ ) are widely used in the treatment of different pathologies and in diagnostics[44]. In particular, $1 \mu \mathrm{m}$ discoidal particles have been previously proposed as an innovative drug delivery platform for cancer therapy and imaging, as extensively reported in the literature[45, 46]. Moreover, although $3 \mu \mathrm{m}$ spherical particles are unlikely to be administered intravenously, they have been used here to test our in vitro system and to obtain a proof of concept demonstration of how size affects particle's margination. The velocity profiles, the capillary radial distribution and marginationof the chosen $\mu$-Pswere studied in physiologically relevant flow conditions. Finally, we validated the findings of ourin vitrostudy by performing intravitalconfocalmicroscopyof the same $\mu$-Psin the microcirculation of live mice. Ourresults provide the first quantitative analysis of the effect of bloodflow on margination of microparticlesin the presence of RBCs.

\section{Materials and methods}

\section{$2.1 \mu-P$ synthesis and characterization}

Synthesis.poly(lactic-co-glycolic acid (PLGA) $\mu-P s$ (Figure A1 and A2) (PLGA ester terminated (lactide/glycolide $=50: 50$ ) viscosity range 0.95-1.20 dl from LACTEL (Pelham, Alabama, USA) were prepared by a modified S/O/W emulsion method as mentioned in our previous studies [47]. In summary, PLGA (50:50) was dissolved in dichloromethane (DCM) to form a 5\% w/v PLGA/DCM solution. The organic phase was mixed with poly vinyl acid(PVA), $2.5 \% \mathrm{w} / \mathrm{v}$ by vortex mixing and sonication (3000 rpm) for 3 minutes. The mixture was gradually dropped into a solution of PVA1\% $\mathrm{w} / \mathrm{v}$. The resulting suspension was stirred with a magnetic stir bar for $6 \mathrm{~h}$ and the DCM was eliminated by evaporation. Subsequently, the suspension was washed three times by centrifugation at $5000 \mathrm{rpm}$ for 10 minutes. $\mu$-Ps were filtered by MF-Millipore ${ }^{\mathrm{TM}}$ Membrane Filters at $3 \mu \mathrm{m}$ and 1 $\mu \mathrm{m}$ to obtain a homogenous size distribution then freeze-dried and stored as well at $-80^{\circ} \mathrm{C}$. PLGA is a copolymer already approved by FDA and widely used for several pre-clinical applications as material for drug delivery vector [48-50]. 
Discoidal multi-stage vectors (MSV)(Figure B1 and B2) weremicrofabricatedas previously reported[51]. We produced arrays of $2 \mathrm{~mm}$ diameter, $300 \mathrm{~nm}$ deep cylindrical trenches into SiN masked, $0.005 \mathrm{~W} \mathrm{~cm}$, p-type silicon wafer by UV photolithography and reactive ion etch. The trenches wereselectively porosified by electrochemical etching of the patterned substrate in $\mathrm{HF}$ ethanoic solution, first applying a porosification current profile and then increasing the current to form a highly porous layer at the particle/substrate interface to enable particle release. The solution composition and porosification current density depends on the target pore size and porosity for each specific particle and is detailed elsewhere[51]. All particles were thoroughly rinsed in deionizedwater multiple times to ensure removal of processing reagents. Surface modification of MSV was achieved using established protocols [52]. Rods (Figure C1 and C2) were microfabricatedas reported elsewhere[53]. Briefly, a heavily doped P-type (100) wafer with a resistivity of $0.005 \mathrm{ohm}-\mathrm{cm}$ (Silicon Quest, Inc, Santa Clara, CA, USA) was exposed to 1:3 HF (49\%): ethanol solution and electrochemically etched with applied current density of $7 \mathrm{~mA} / \mathrm{cm} 2$ for $65 \mathrm{sec}$ or $125 \mathrm{sec}$ to form a $200 \mathrm{~nm}$ or $400 \mathrm{~nm}$ thick porous silicon layer, respectively. The current density was then increased to $76 \mathrm{~mA} / \mathrm{cm} 2$ and applied for $6 \mathrm{sec}$ to form a second porous layer, the high porosity release layer. The double-layer films were washed and spin-dried.
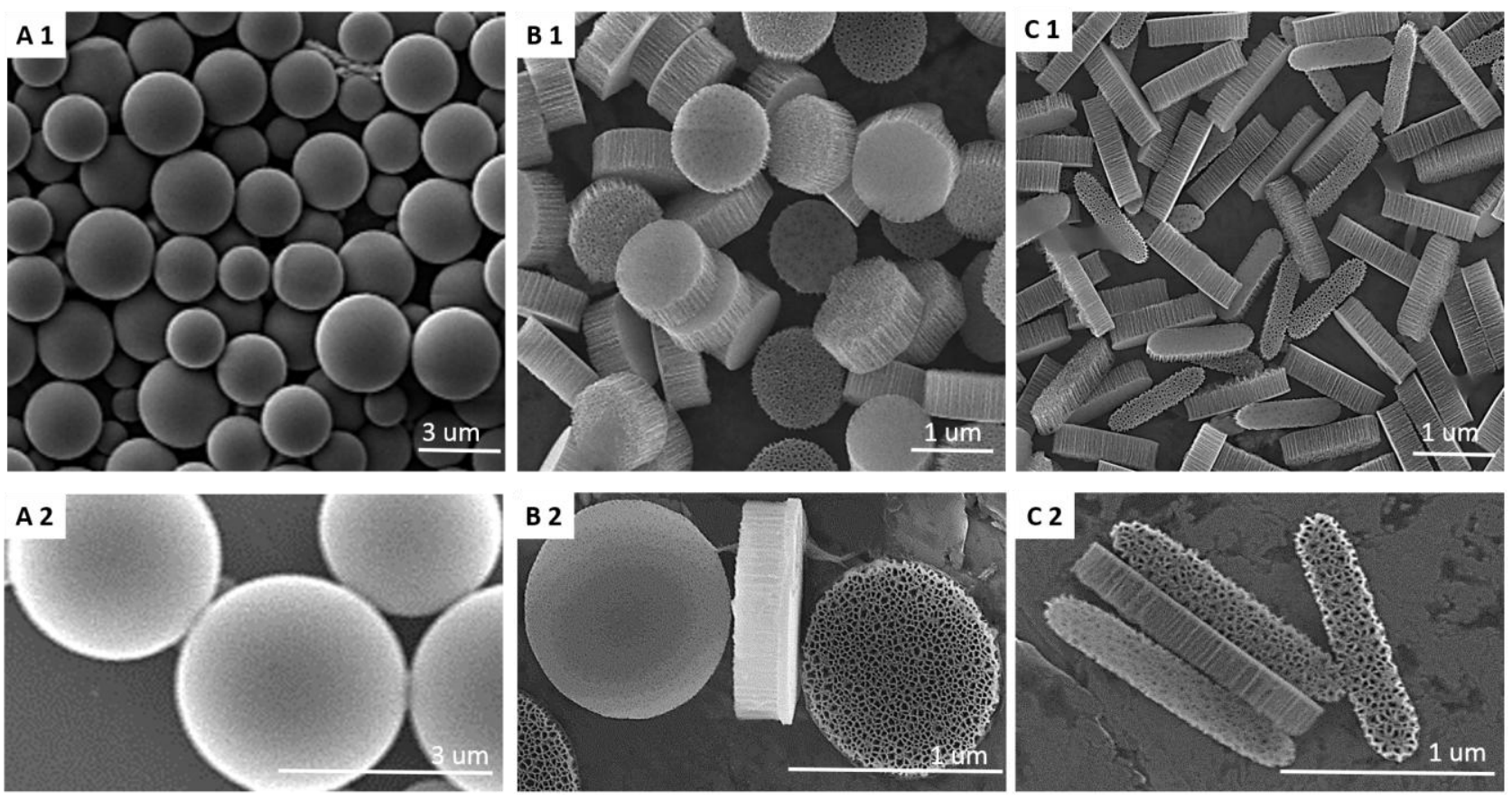

Figure 1: Scanning electron micrographs of micro-particles. Spherical PLGA particles at low (A1) and high (A2) magnification. Discoid multi-stage vectors at low (B1) and high (B2) magnification. Rods at low (C1) and high (C2) magnification. All particle types have a high degree of uniformity in terms of size, shape and surface features.

A 40-nm thick low-temperature oxide was deposited on the porous film in an LPCVD furnace. The photolithography process was carried out using an i-line contact aligner (K.Suss MA6 mask aligner) and NR9-250P photoresist (FuturrexInc., Franklin, NJ, USA). A dark-field photomask (Chrome on quartz) consisting of a pattern array of $1900 \mathrm{x} 400 \mathrm{~nm}$ rectangles with $2500 \mathrm{x} 1000 \mathrm{~nm}$ pitch was obtained from HTA Photomask (San Jose, CA, USA). A thin NR9-250P photoresist layer was spun on the double-layer film at 3000RPM and baked. Exposure time was optimized to obtain a $1500 \mathrm{x}$ $200 \mathrm{~nm}$ pattern on 200-nm thick film. The patterns were transferred into the double-layer porous films by dry etch in CF4 plasma (PlasmathermBatchTop, 15sccm CF4, 100 mTorr, 200W RF). 
After striping the low-temperature oxide in dilute HF, the porous silicon particles were washed while retained on the substrate, and then released by ultrasound in isopropyl alcohol (IPA).

It should be pointed out that fluid dynamic interactions between microparticles and RBCs are not dependent on the specific material of the microparticles in our experimental conditions. Indeed, buoyancy effects coming from the density difference between microparticles and suspending fluids are negligible given microparticles size and the time scale of the flow experiments (e.g., in a typical

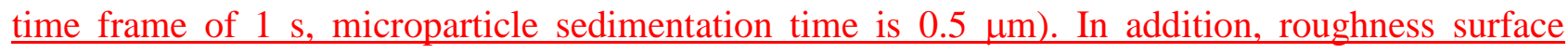
differences (smooth vs porous) can be also neglected as far as hydrodynamic interactions are concerned, since the latter are dominated by collisions between microparticles and RBCs. Finally, the (negative) surface charge is in the same range for all the microparticles (see Table 1).

Table 1: Summary of the relevant chemical and physical properties of themicro-particles used in this study

\begin{tabular}{|l|c|c|c|c|}
\hline & Small spheres & Big spheres & Discs & Rods \\
\hline Size (D x h) & $0.9 \pm 0.635 \mu \mathrm{m}$ & $3 \pm 0.965 \mu \mathrm{m}$ & $1 \mu \mathrm{m} \times 400 \mathrm{~nm}$ & $400 \mathrm{~nm} \times 1.8 \mu \mathrm{m}$ \\
\hline Shape & Spherical & Spherical & Plateloid & Cylindroid \\
\hline Volume & $0.3 \mu \mathrm{m}^{3}$ & $14.13 \mu \mathrm{m}^{3}$ & $0.3 \mu \mathrm{m}^{3}$ & $0.3 \mu \mathrm{m}^{3}$ \\
\hline Porosity & None & None & $\sim 60 \%$ & $\sim 60 \%$ \\
\hline Z-potential & $-40.2 \pm 0.3 \mathrm{mV}$ & $-52.7 \pm 1.5 \mathrm{mV}$ & $-17.7 \pm 3.3 \mathrm{mV}$ & $-18.4 \pm 3.1 \mathrm{mV}$ \\
\hline $\begin{array}{l}\text { Z-potential } \\
\text { labeled-particles }\end{array}$ & $-35.2 \pm 0.3 \mathrm{mV}$ & $-32.7 \pm 2.5 \mathrm{mV}$ & $-20 \pm 1.2 \mathrm{mV}$ & $-19 \pm 0.6 \mathrm{mV}$ \\
\hline
\end{tabular}

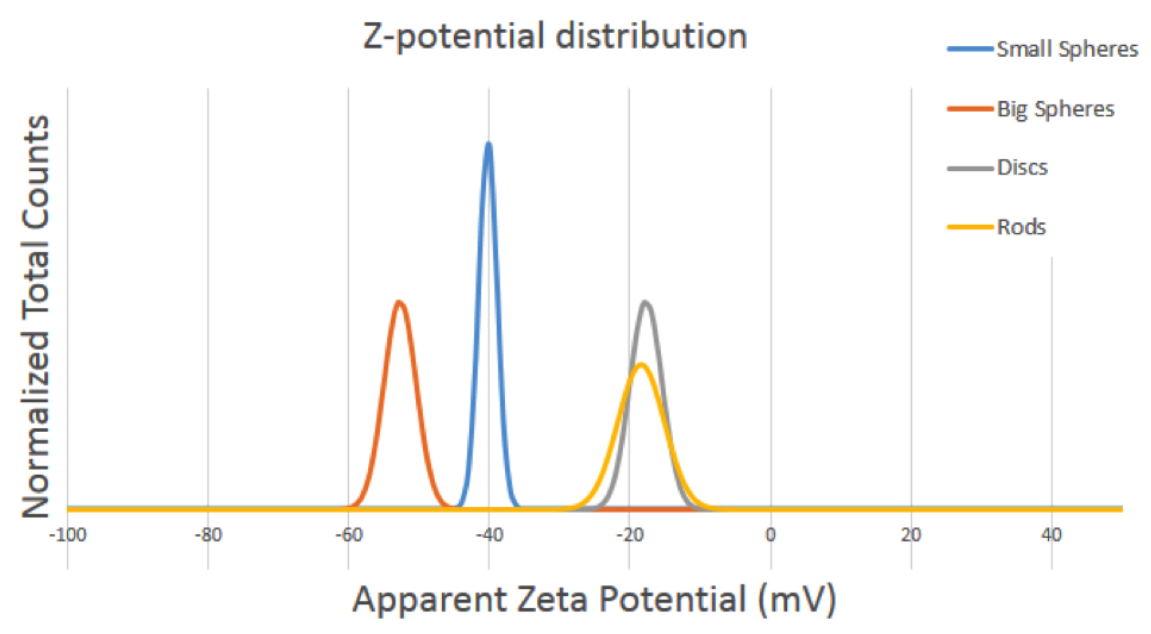

Label conjugation for in vivo studies. Targeted fluorescent particles were generated by sequential chemical modification using a protocol modified from [54]. In brief, the surface of oxidized particles was aminated using 1\% (v/v) 3-aminopropyltriethoxysilane solution (APTES; SigmaAldrich, St. Louis, MO, USA) in 98\% isopropanol (IPA; Sigma-Aldrich) for $2 \mathrm{~h}$ at $35^{\circ} \mathrm{C}$. Aminated particles were washed twice in IPA, dried under vacuum overnight to polymerize the silane layer, and then reacted with $1 \mathrm{mg} / \mathrm{ml}$ Alexa Fluor 555 succidymal ester (Life Technology, Carlsbad, CA, USA) suspended in dimethyl sulfoxide (DMSO; Sigma-Aldrich) containing 100mMtriethanol amine (Sigma-Aldrich). Rhodamine labeled PLGA (Sigma-Aldrich) was synthesized with the same procedure with the addition ofRhodamine A (Sigma Aldrich) and mixed at the PLGA solution at a concentration of $10 \mu \mathrm{g} / \mathrm{ml}$. 
Zeta potential. The zeta potential of the $\mu$-Ps was determined using a Zetasizer(ZEN3600, Malvern, Worcestershire, UK). $2 \mathrm{ml}$ of $\mu-\mathrm{P}$ suspension were injected into a sample cell counter filled with phosphate buffer (1.4 ml, pH 7.3). The cell was sonicated for 2 minutes, and then an electrode probe was placed in the cells. Measurements were performed at room temperature in triplicate.

Size distribution. $\mu-\mathrm{P}$ size distribution and count was obtained using a Z2 Coulture Particle Counter and Size Analyzer (Beckman Coulter, Fullerton, CA, USA). $10 \mathrm{ul}$ of the samples were dispersed in the balanced electrolyte solution (ISOTON II Diluent, Beckman Coulter Fullerton, CA, USA) and sonicated for 5 seconds to ensure a homogenous dispersion.

Scanning electron microscopy. Samples were washed twice in deionized water to remove salt, placed on aluminum mounts (Ted Pella, Redding, CA, USA), and left in a vacuum dessicator to dry overnight. Samples were examined with a Zeiss Neon 40 microscope equipped with an in-lens detector at an acceleration voltage between 5 and $10 \mathrm{keV}$ at a working distance of approximately $5 \mathrm{~mm}$.

\subsection{In vitro experiments}

Blood samples. Fresh venous blood samples were drawn from healthy donors and diluted to a concentration of $10 \%$ by volume with $\mathrm{ACD}(0.6 \%$ citric acid, $2.3 \%$ sodium citrate, $1.1 \%$ anhydrous dextrose and $96 \%$ water) as anticoagulant and bovine serum albumin. All samples were used within $4 \mathrm{~h}$ of collection. The concentration of $10 \%$ was selected to match the hematocrit value (Hct), i.e., the percentage of cell volume with respect to total blood volume in microcirculation (10 to 26\%)[55]. The higher values of Hct (around 45\%) that are found in routine blood tests are associated with large vessels, whereas Hct decreases going from the macro- to the microvasculature. This is due to entrance effects and the inhomogeneous radial distribution of RBCs that tend to concentrate along the vessel centerline (Fahraeus effect [56]).

$\mu$-Ps and RBCs/ $\mu$-P suspension. Two different suspensions of each cell type were used: 1) a $\mu$-P suspension, Hct $=0 \%$, used as control, consisting of $\mu$-Ps suspended ( $0.004 \%$ weight $)$ in ACD andbovine serum albumin( $1 \mathrm{~g}$ in $5 \mathrm{ml}$ of $\mathrm{ACD}$ ); 2) a $\mu$-P/RBC suspension, Hct=10\%, consisting of $\mu$-Ps $(0.004 \%$ weight $)$ in a RBC suspension with $\mathrm{Hct}=10 \%$.

Experimental apparatus. Images of the flowing suspensions wereacquired witha high-speed video camera (Phantom 4.3, operated up to 1000 frames/s) using a high magnification oil immersion objective $(100 \times)$. Since we focus in the capillary midplane, around which the velocity profile is rather flat, velocity variations along the vertical direction are quite small in the optical section of our observations, ensuring a 2D view. The experiments were carried out in $50 \mu \mathrm{m}$ diameter silica microcapillaries (Polymicro Technologies, Phoenix, AZ, USA) placed on the motorized x-y stage (Ludl, Hawthorne, NY, USA) of an inverted microscope (Zeiss Axiovert 100) equipped with a motor assembly for remote focus control (Ludl, Hawthorne, NY, USA). The capillary tube was connected to a micro-pumping system loaded with the $\mu$-P/RBC suspension. The $50 \mu \mathrm{m}$ silica capillary mimics the hydrodynamic conditions of the human microcirculation, such as laminar flow and physiological pressure $(20-60 \mathrm{mmHg})$ [57].All measurements were performed in the midplaneof the capillary (length $5 \mathrm{~cm}$ ) at the imposed physiological pressure of $20 \mathrm{mmHg}$. The 
tracking procedure consists in selecting a particle in the image at some time point and then finding its subsequent positions manually frame by frame. At each position the particle is marked by a cross in the image overlay in order to facilitate the identification of the next position. Given the small particle size the positioning error is limited to a few pixels only. The particle $\mathrm{x}$ and $\mathrm{y}$ coordinates are stored in a file by the image analysis software used in our work. Particle concentration is quite low, so particle-particle interactions are negligible.The so-obtained data were corrected using Snell's law [58] to account for the optical artefact due to the difference in refractive index of glass ( $\left.\mathrm{n}_{\mathrm{g}} \sim 1.5\right)$ and suspending liquid $\left(\mathrm{n}_{\mathrm{sl}} \sim 1.33\right)$, which caused an apparent shift of $\mu$-Ps and RBCs position towards the tube centerline.

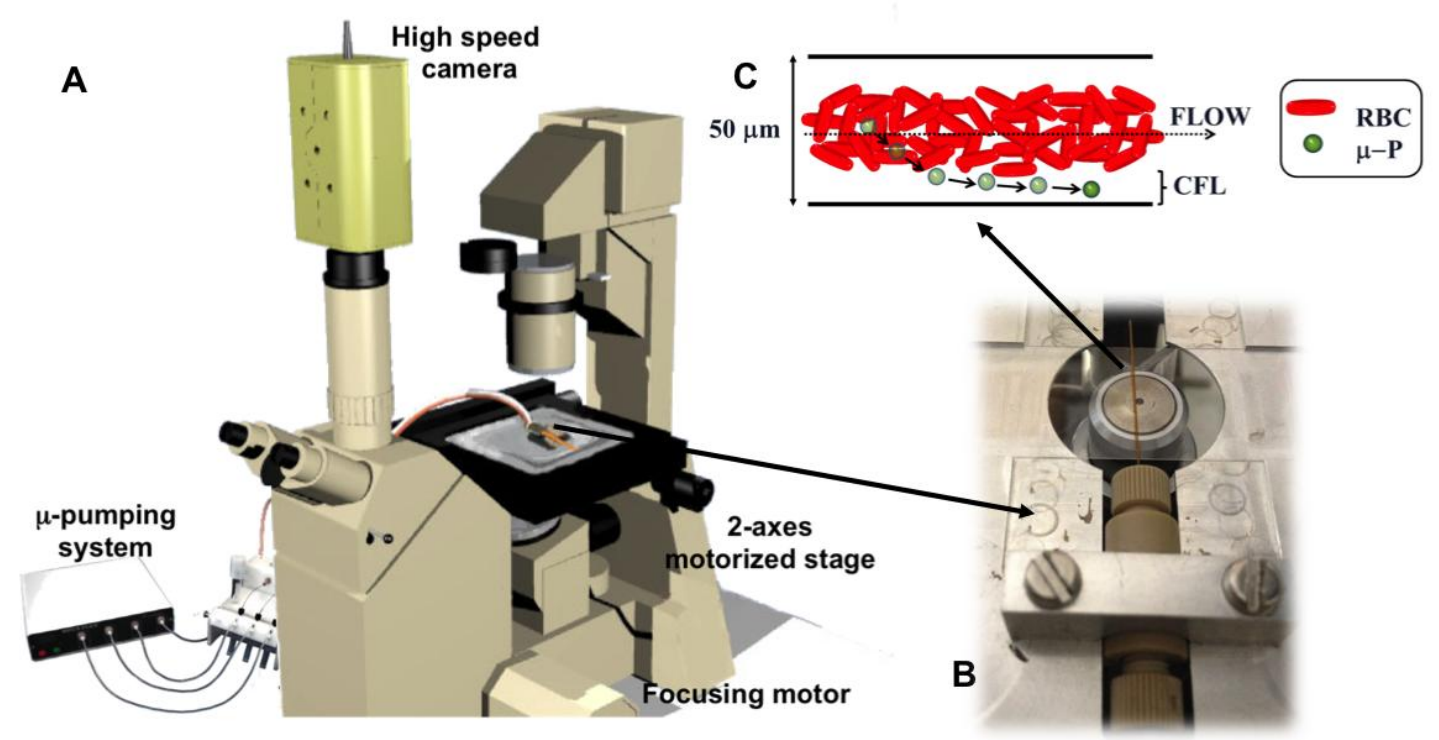

Figure 2: A.Scheme of the experimental apparatus; B. Zoom on the $50 \mu \mathrm{m}$ glass microcapillary tube; C.Cartoon of $\mu$ Pmargination in presence of RBC flow inside the microcapillary.

\subsection{Intravital experiments}

All animal experiments were approved by The Houston Methodist Institutional Animal Care and Use Committee guidelines (Houston, TX, USA) and were performed in accordance and under IACUC-approved protocols AUP 0611-0032.

Evaluation of particle flow dynamics. $\mu-\mathrm{P}$ flow and the respective interactions with vessel walls were assessed by intravitalconfocal microscopy. Transgenic Tie2-green fluorescent protein (GFP) mice (5-6 weeks of age) specifically expressing GFP on the vascular endothelium were used to visualize the flow dynamics of labeled microparticles. For intravital confocal microscopy imaging, skin vessels were exposed using a skin-flap procedure as previously described [53].Briefly, a midline abdominal incision was made and a wetted cotton applicator was used to peel away the skin taking care to avoid damaging the superficial vessels. Anesthetized animals were placed on an upright Nikon A1R MP-ready laser scanning confocal microscope platform equipped with a resonance scanner, anisoflurane anesthesia system, heated stage, and custom coverslip mounts [53]. Superficial skin vessels measuring between $40-60 \mu \mathrm{m}$ in diameter were chosen to recapitulate the experimental settings used for in vitrotesting. Labeled particles $\left(1 \times 10^{6}\right.$ MSVs or $5 \times 10^{5}$ PLGA) were injected through the retro-orbital route. The flow of $\mu$-Ps was acquired with the following settings: GFP expressed on the vessel walls was excited at $488 \mathrm{~nm}$ while labeled particles wereexcited at $561 \mathrm{~nm}$ collected at band-pass filters widths $30-50 \mathrm{~nm}$ centered at $525 \mathrm{~nm}$ for GFP- 
labeled particles and at $579 \mathrm{~nm}$ for labeled particles. The analysis of images and movies as well as post-production were performed using Nis-elements software. The total number of animal used were 3 for experimental condition.

\subsection{Statistical Analysis}

All the data are the result of a minimum of three independent experiments on each type of particles. Statistics were calculated with Prism GraphPad software (LaJOlla, California, USA). The significance was calculated through OneWayAnova method, followed by Dunnett's test or with independent Student's T-Test.

\section{Results and discussion}

\subsection{Velocity profilesdemonstrate $R B C$-induced shear trapping of $\mu$-Ps}

Tostudythe effect of RBCs on $\mu-\mathrm{P}$ flow and marginationin vitro, we determined velocity profiles (i.e., the change of velocity of the fluid along the radius of the tube), and the radial distribution of $\mu$ Ps of the Hct $0 \%$ and Hct $10 \%$ suspensionsusing high-speed video.The velocity of a Newtonian fluidin a tube changes from zeroat the tube wall, due to the no-slip condition, to a maximum at the centre of the tube, that results in a parabolic velocityprofile. This is described by the Poiseuille law for steady flow in tubes $\Delta P=\frac{8 \mu L Q}{\pi r^{4}}$ ), where $\Delta \mathrm{P}$ is the pressure losses, $\mathrm{L}$ and $\mathrm{r}$ are respectively the capillary length and radius, $\mathrm{m}$ is the suspending fluid viscosity and $\mathrm{Q}$ is the flow rate. This formula appliesonly under laminar flow conditions characterized by smooth streamlines and highly ordered motion. These phenomena are typical ofthe microcirculation, where due to the small size of arterioles, venules and capillaries, the Reynolds number is well below the transition to turbulent flow $[59,60]$.

In this work, velocity profiles wereobtained by tracking the positions of $\mu$-Ps and RBCsalong the capillaryaxisat differentradii(measured from thecenterline). The velocity of $\mu$-Ps or RBCs was determined by plotting their displacement as a function of time. As expected, these plots were linear and the slope was taken as the particle/cell velocity.

$\mu$-Pvelocityis shown in Figure 3 as a function of the distance from the centerline for the four types of $\mu$-Ps without RBCs(Hct 0\%, Figure 3A) and in the presence of RBCs (Hct 10\%, Figure 3B). The continuous line represents the parabolic profile of the fluid velocity and was calculated by applying thePoiseuillelawat $20 \mathrm{mmHg}$, with a viscosity of 1 cPoise, a tube length of $5 \mathrm{~cm}$ anda capillary diameter of $50 \mu \mathrm{m}$ (i.e., with no fitting parameters). The pressure drop in the microcapillarywas taken to be equal to the pressure drop imposed by the micro-pumping system, considering the other pressure losses (e.g., those in the plastic feed tubing)negligible.As shown in Figure 3A, in the absence of RBCs, all data superimposed the calculated Poiseuille profile, irrespective of particle type. This resultwasattributed to the low concentration and small size (compared to the tube diameter)of the $\mu$-Ps, which just follow the motion of the fluid. 

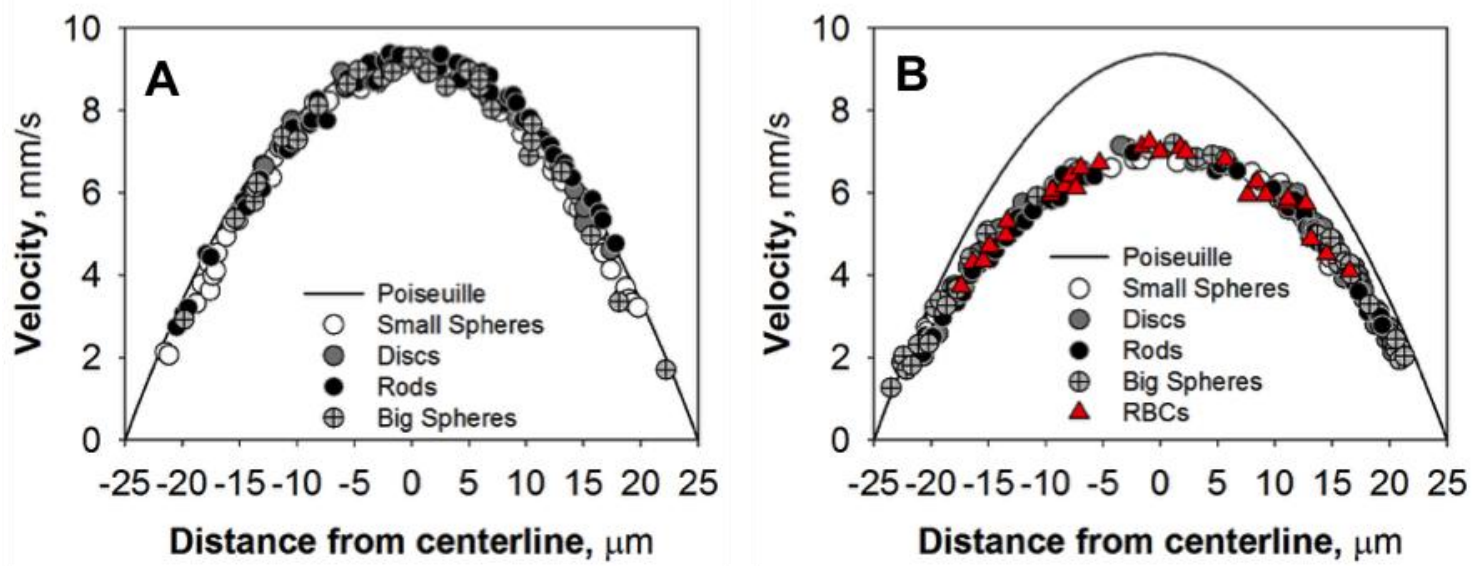

Figure 3: A. $\mu$-P velocity profile at $20 \mathrm{mmHg}$ for small spheres $(1 \mu \mathrm{m})$ (white circles), big spheres $(3 \mu \mathrm{m})(\operatorname{crossed}$ circles), disc (grey circles) and rods (black circles); B. $\mu$-Ps and RBCs (red triangles) velocity profile at $20 \mathrm{mmHg}$. Each point represents $\mathrm{RBC} / \mu$-Ps velocity. The solid lines represent the suspending fluid velocity as calculated from the Poiseuille tube flow.

A remarkable difference in velocity profiles occurred when RBCs were suspended in the fluid at Hct $10 \%$.As shown in Figure 3B, RBC velocity (red triangles) was lower than the calculated velocity of the suspending fluid in the absence of RBCs (continuous line). This reduction was due to the increment of suspension viscosity brought about by the RBCs. A similarvelocity decrease was reported fordeformable objects, such as liquid vesicles [61, 62] and capsules [61, 63, 64], which are considered model systems of RBCs. When $\mu$-Psflowed together with RBCs, the former displayed the same velocity profile as the latter. This is shown in Figure $3 \mathrm{~B}$ where all the data are superimposed irrespective of $\mu-\mathrm{P}$ size or shape. In other words, RBCs govern the flow behavior of the suspension, and their effect on $\mu$-Ps can be referred to as "shear trapping" sincethe particle motion along the flow was slower than in the absence of RBCs.

\section{$3.2 \mu$-Ps are marginated by RBCs in the cell-free layer}

To quantify the radial distribution of the $\mu$-Ps, the center plane of the capillary was divided in 50 layers, each accounting for $1 \mu \mathrm{m}$ (i.e., a dimension similar to particlessize). To improve the statistics, the number of $\mu$-Ps was averaged between pairs of layers symmetric to the centreline(e.g., the layer between -10 and $-9 \mu \mathrm{m}$ and the layer between 9 and $10 \mu \mathrm{m}$ ). When we plotted the percentage of $\mu$-Ps per layer (i.e., the number of $\mu$-Ps in a layer divided by the total number of $\square$ Ps) as a function of capillary radiusfor the small sphere $\mu-\mathrm{P}$ (Figure 4), there was aparabolicdistribution along the radial directionthat resembled the $\mu$-P velocity profile. This distribution was due to the fact that the number of $\mu$-Ps that travelled across the field of view in a given time intervalwas higher in the center of the capillary than near the wall because of the difference in particle velocity. In fact, when we normalized with respect to the number of particles in each layer with the corresponding $\mu-\mathrm{P}$ velocity, the distribution was uniform (Figure 4, inset), which shows that the concentration of the $\mu$-Ps along the capillary radius was constant over the entire cross section. These resultsarein line with previous results obtained atlow Reynolds numbers and at short distance from the capillary entrance [65], and were applicable to all the types of particles considered. 


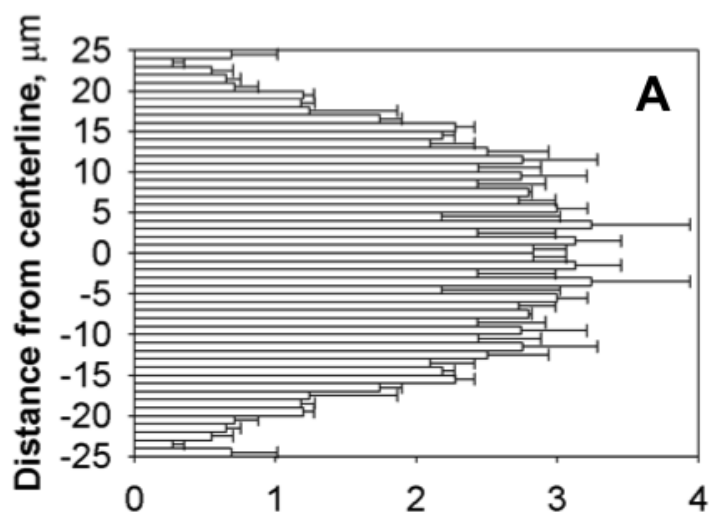

Small Sphere percentage, \%

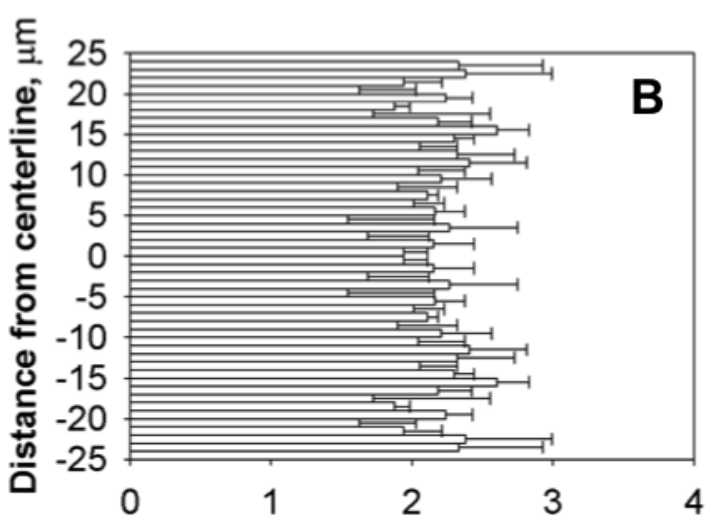

Small Sphere percentage, \%

Figure 4: A.Distribution profile of $\mu$-Ps across the capillary in the absence of RBCs (Hct $0 \%$ ) at $20 \mathrm{mmHg}$ : B. Distribution profile of $\mu$-Ps normalized with respect to particle velocities. The errorbarsrepresent the standard deviation.

When RBCs are flowing in a capillary, a cell-free layer is formed near the wall (see Figure 5).In our conditions (Hct 10\%, pressure drop $20 \mathrm{mmHg}$ ), the cell-free layer was located about $6 \mu \mathrm{m}$ from the wall.

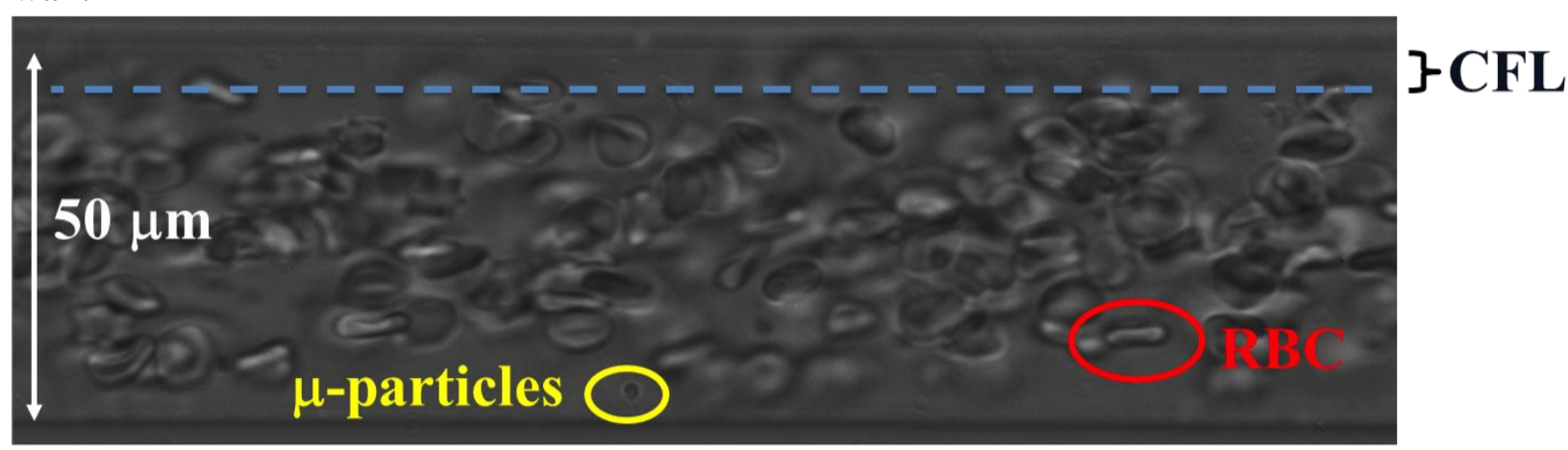

Figure 5: Image of the flow of $\mu$-Ps and RBCs inside a 50- $\mu \mathrm{m}$ microcapillary. The migration of RBCs towards the capillary center-line leaves a cell-free layer (CFL) of $6 \mu \mathrm{m}$.

The radial distribution of $\mu$-Ps changed remarkably in the presence ofRBCs. In fact, in this case,all the $\mu$-Ps accumulated near the wall (see Figure 6). When flowed with RBCs, $\mu$-Psmarginatedsimilar to platelets and white blood cells. The peak accumulationwaslocated at $\pm 19 \mu \mathrm{m}$ from the capillary axis, which corresponded to the edge of the cell-free layer (Figure 5). These results arein line withrecent numerical simulations in which platelet accumulation peaked just outside the RBC central core[66].We measured the thickness of the cell-free layer using image analysis, and obtained a value of $6 \mu \mathrm{m}$, which was in very good agreement with results from numerical simulations in similar flow conditions [35, 67].All particle types showed the same behavior, but the percentage of marginated $\mu$-Ps (i.e.,the ones in the cell-free layer) was clearly dependent on size and shape. A similar increase of near-wall concentration has been reported for platelets and white blood cells in capillary flow $[24,68,69]$. 

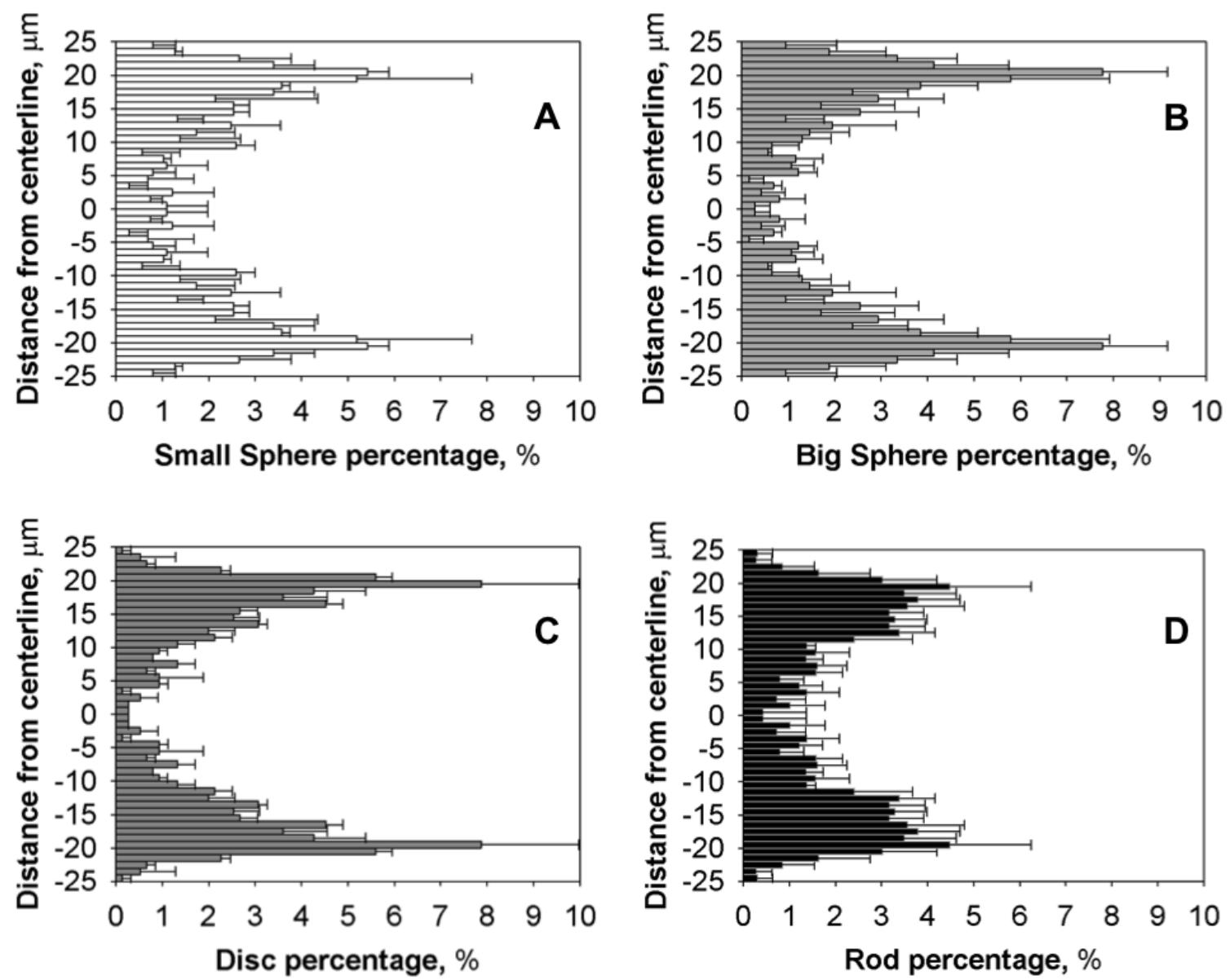

Figure 6: Distribution profiles of $\mu-\mathrm{Ps}$ alongthe capillary radius in the presence of RBCs (Hct $10 \%)$ at $20 \mathrm{mmHg}$. A.Small spheres $(1 \mu \mathrm{m})$; B.Big spheres $(3 \mu \mathrm{m})$; C.Discs and D.Rods. The error bars represent the standard deviation.

To quantify and compare the effect of particle size and shape on $\mu-\mathrm{P}$ margination, the percentage of marginated particles was defined as the fraction of particles in a $6-\mu \mathrm{m}$ thick near-wall layer (i.e., the cell-free layer). Figure 7A shows the percentage of margination of spherical particles with different diameters $(1 \mu \mathrm{m}$ : small spheres and $3 \mu \mathrm{m}$ : big spheres). Margination increasedsignificantly with particle size, which is in accordance withnumerical studies [33, 35].

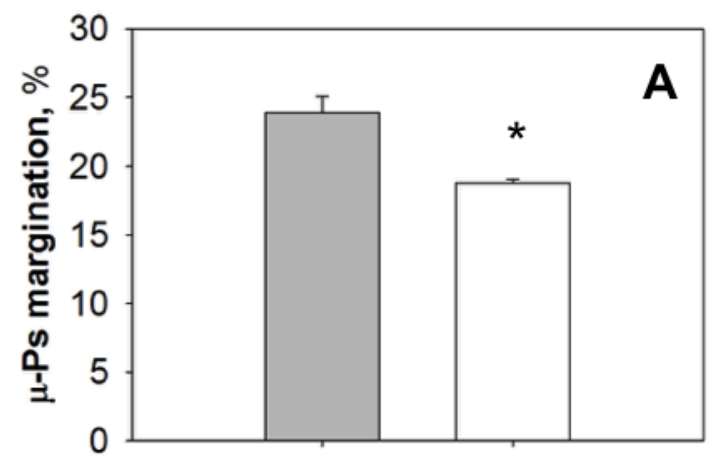

Big Spheres Small Spheres

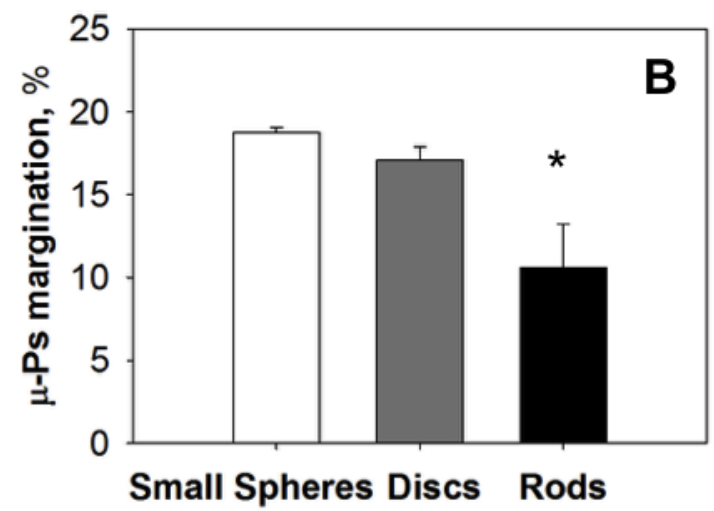

Figure 7: Percentage of marginated $\mu$-Ps in the presence of RBCs (Hct 10\%) at $20 \mathrm{mmHg}$. A. Comparison between particles of the same shape butdifferent size. There is a significant difference between the two sets of data $(\mathrm{P}=0.005)$; B. Comparison ofparticles of the same size but different shape. While the difference between disc-shaped and small spheres is not significant, the difference between rod-shaped and small spheres is significant $(\mathrm{P}=0.006)$. 
The effect of particle shape (spheres, rods and discs) was evaluated for $\mu$-Ps withsimilarhydrodynamic volume(Table 1). As shown in Figure 7B, spheres and discs had a comparable margination propensity, while margination of rod-shaped particles was significantlylower (statistically significant: $\mathrm{P}=0.006$ ). Recent numerical studies suggestthat discoid particles are the most efficient carriers for targeted drug delivery because of their higher adhesion properties due, in turn, to the larger contact area versus spherical carriers[53, 70].

\section{3. $\mu$-P margination in vivo confirms in vitro results}

The flow behavior of $\mu$-Ps was investigated in vivousing intravital confocal microscopy. All the 4 types of particles listed in Table 1 weretrackedinmicrocapillaries with diameters close to $50 \mu \mathrm{m}$ (i.e., the diameter ofthe capillaries used in the in vitroexperiments). The intensity profile graphs (Figure 8) were obtained analyzing the in vivo images; each peak indicates increased fluorescence intensity above a line linking the vessel walls. Green peaks and red peaks occur in correspondence of vessel walls and $\mu-P s$, respectively. Representative in vivo images are shownbelow the graphs; red dots indicate the rhodamine-labeled $\mu$-Ps. The graph and the images illustrate that the accumulation of rod-like particles near the vessel walls was less pronounced than that of the other types of $\mu$-Ps.
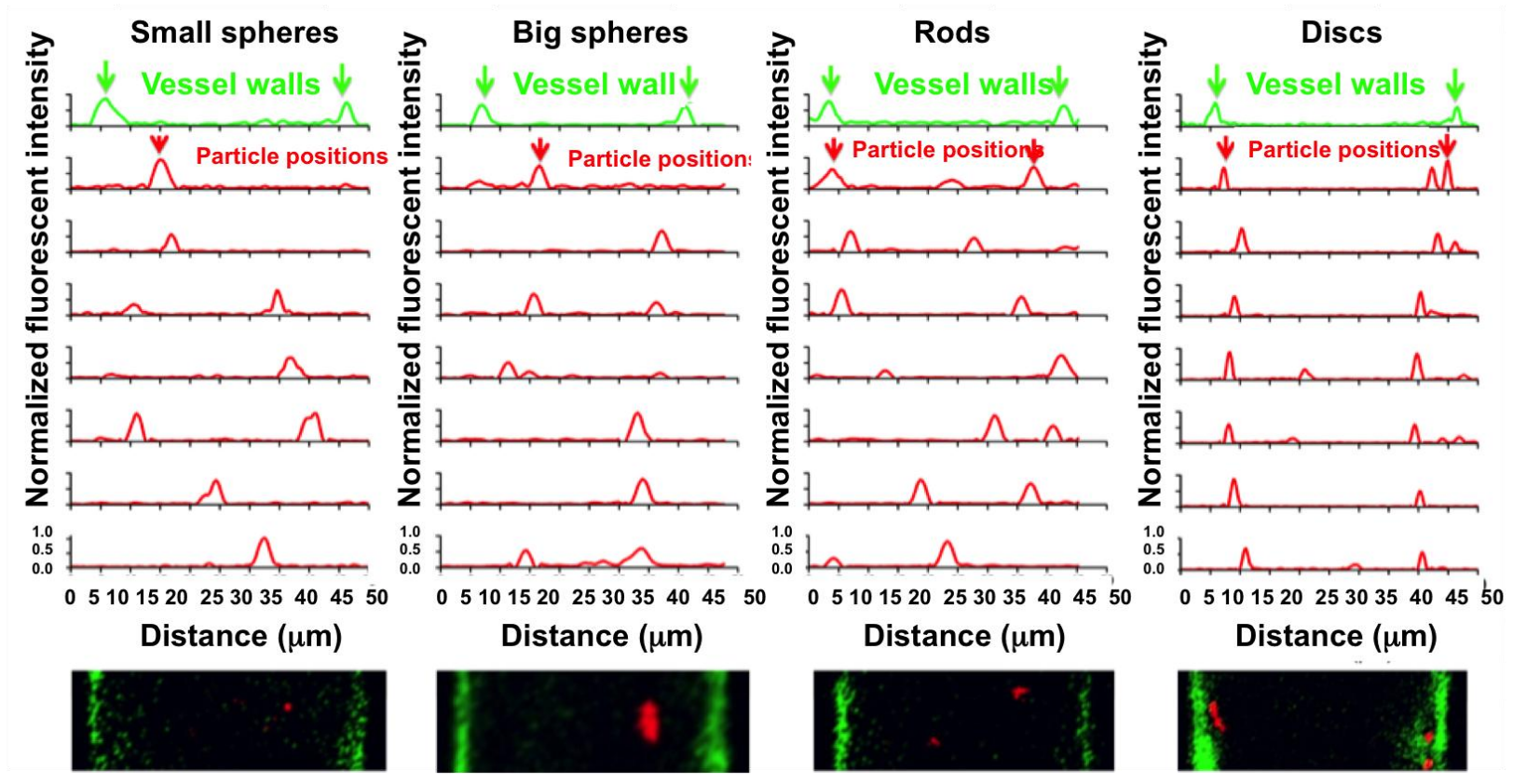

Figure 8: Intensity profile graphs obtained from intravitalmicroscopy imagesof micro-particles (red) in mice microvasculature. Below are representative frames isolated from the intravital micrographs of the injected microparticles in blood vessels measuring 50 micron in diameter. The vessel walls are green due to the GFP expressed by the endothelial cells of the transgenic Tie2-GFP mice.

To quantify $\mu$-P margination, we conducted the same image analysis of the in vitro experiments on a statistically relevant number of images (see Figure 9). These data show that $\mu$-Ps tend to accumulate near the vessel walls, in a size- and shape-dependent fashion. The near-wall accumulation of big $\mu$-Ps $(3 \mu \mathrm{m})$ was greater that of small $\mu-P s(1 \mu \mathrm{m})$. In terms of shape, small spheres and discoidal $\mu$-Ps had a similar margination propensity, while margination of rods was significantly lower $(\mathrm{P}=0.023)$ compared with the other two shapes. These observations confirm our 
in vitrofindings. This trend is immediately comprehensible in Figure 9.C in which the movie frames acquired by intravital microscopy were merged to obtain a dispersion map of $\mu-\mathrm{P}$ flow into mouse capillaries.
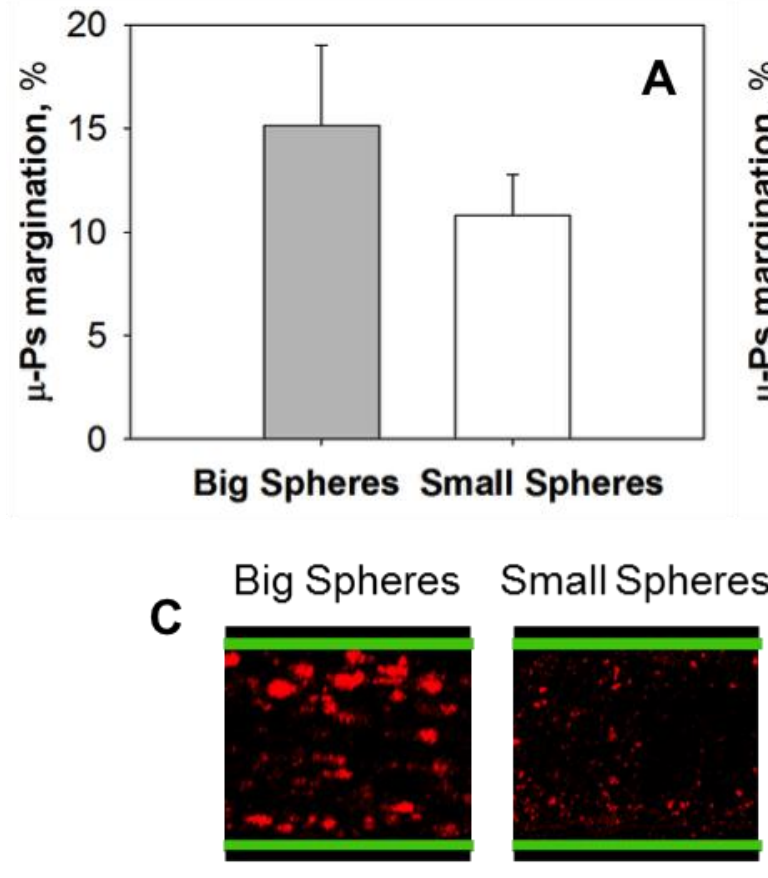
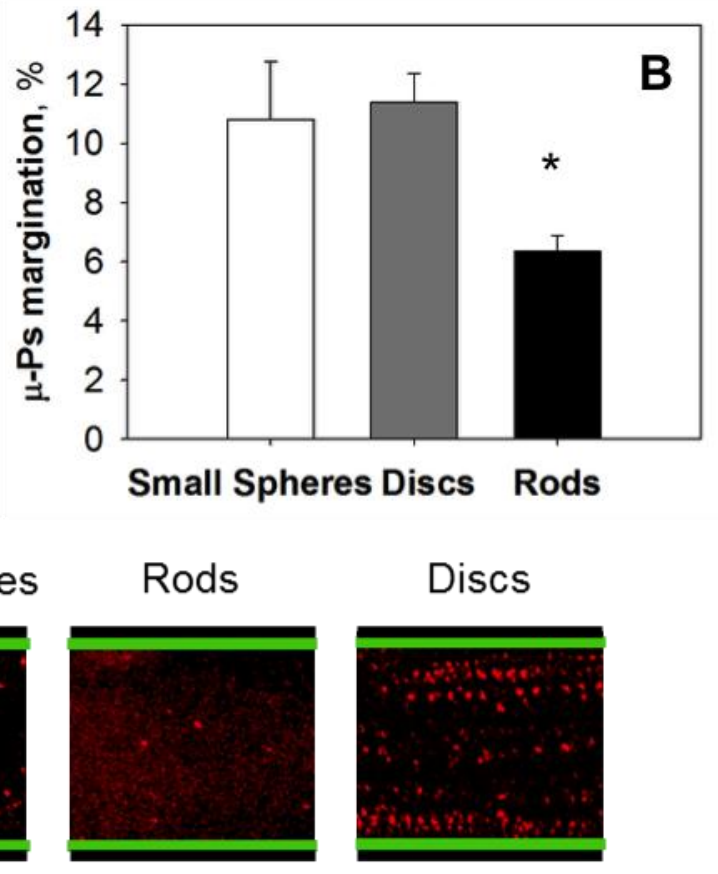

Figure 9: Percentage ofmarginated $\mu$-Ps in the presence of RBCs (Hct 10\%) at $20 \mathrm{mmHg}$ by intravital microscopy. A. Comparison between particles with the same shape and different size: the difference between smalland bigspheres is not significant. B. Comparison among particles with the same volume and different shapes. Here, while the difference between discs and small spheres is not significant, whereas the difference between rods and discs is significant $(\mathrm{P}=0.023)$. C. 9.2: Dispersion map obtained merging thetime frames of a movie acquired usingintravital microscopy while $\mu$-Ps were flowing into the vessels (capillarywalls are shown in green and $\mu$-Ps in red).

Figure 10 shows the tracks of the particlesobtained by overlapping multiple frames of an intravital microscopy movie (big spheres and discs are shown as representative). Considering the image obtained by merging the frames as a Cartesian graph and projecting $\mathrm{x}$ and $\mathrm{y} \mu-\mathrm{P}$ positions as a function of time, we obtained two Cartesian graphs (B and C). B1 and B2 representthe horizontal particle position as a function of time, Cgraphs represent the vertical particle position in function of time.The slope of the lines in panelsB and $\mathrm{C}$ representsthe average horizontal and vertical velocity of particles. From the B graphs, we calculated the horizontal velocity of all four types of particles, and found that big spheres move faster than the others, small spheres move faster than rods and discs and that discs are the slowest. The slope of the segment obtained in B is representative of particle speed. The average horizontal velocity of all four particle types is shown in $\mathrm{D}$. The superior margination of discs correlate to a slower speed. Although rods have a poor margination, theirspeed is slower than that of big and small PLGA spheres; this effect is probably due their shape. 

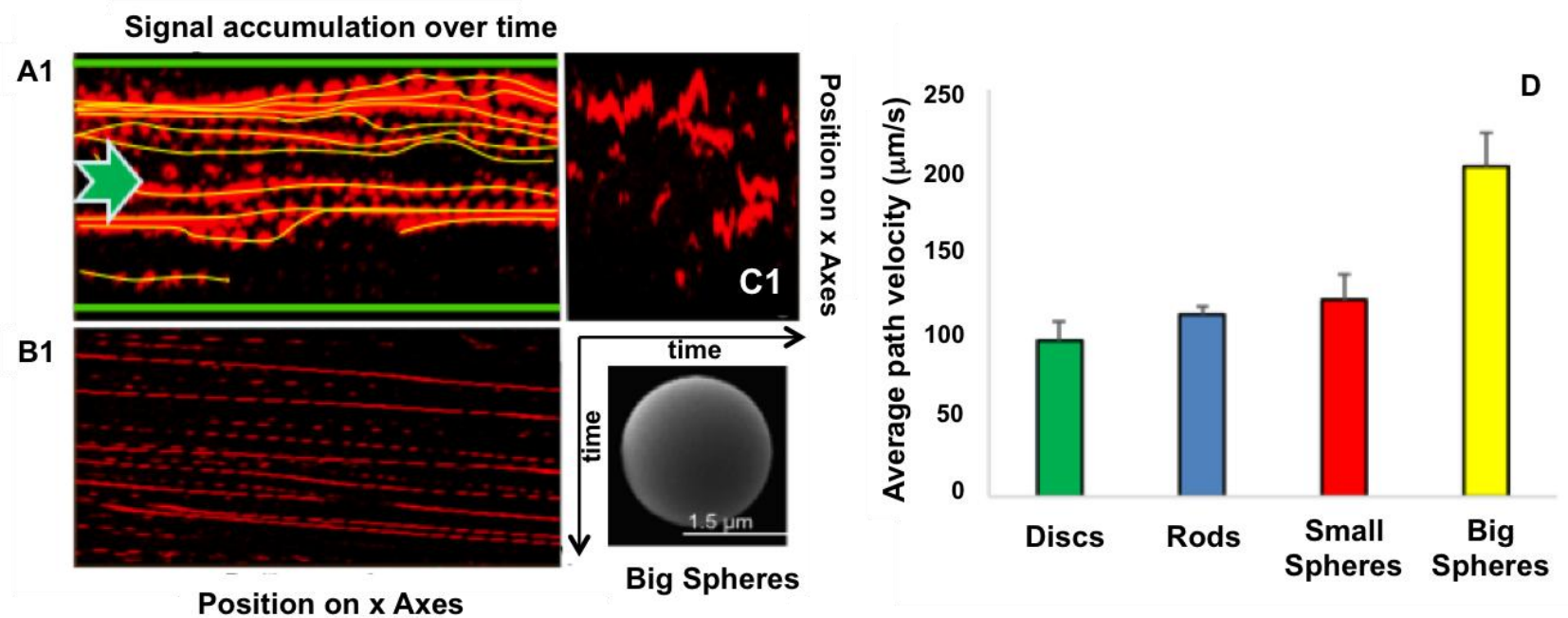

Signal accumulation over time
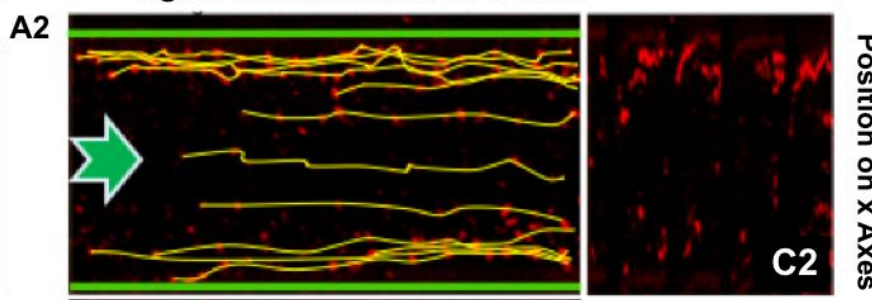

Figure 10: Particle tracks in yellow are shown by overlapping multiple frames from IVM movie (yellow lines) (A). Green arrows represent flow direction. The figure shows particle motion on the $\mathrm{x}$ (B) and y (C) axes over time. These projections represent a Cartesian graph in which one of the axis is the time and the other represents $\mathrm{X}$ or Y position of the particles. The slope of the obtained lines in panel B representsthe average horizontal velocity of particles. (Because of the smaller size of the discs, the lines in B2 were obtained by joining the dots). The vessels walls are represented as green lines. The average horizontal velocity of all particle types is shown in $\mathrm{D}$.

\section{4. $\mu$-P marginationis due to shear-induced collision with RBCs}

The results reported in this study show that $\mu$-Psunder flow in capillaries marginate in the cell-free layer only when RBCs are present. We conclude that $\mu-\mathrm{P}$ marginationoccurred as a consequence of thecollision between RBCs and the particles that triggered a change in the particle trajectory, thereby leading to a non-Brownian diffusion along the radial direction. This shear-induced diffusion acted also on the RBCs and was counterbalanced by the hydrodynamic lift near the wall, thus leading to the cell-free layer. In the case of $\mu$-Ps interacting with RBCs, as soon asa particle reached the edge of the cell-free layerthanks to the shear-induced diffusion, it moved rapidly inside it (waterfall effect) and remained entrappedin this region of the capillary [31, 32]. In fact, once the particlesreached the cell-free layer, shear-induced collisions between particlesbecame less probable due to the low concentration and small size of $\mu$-Ps.Consequently, any further particle radial diffusion was strongly suppressed and $\mu$-Ps accumulated in the cell-free layer, giving rise to the margination effect.This explanation is also consonant with a link between particle size and shape and margination behavior. Concerning size, wefound that $3-\mu \mathrm{m}$ spheres marginatedmore efficientlythandid $1-\mu \mathrm{m}$ spheres - a finding that confirmsa numerical study that the optimal dimension for the best margination is $2-3 \mu \mathrm{m}[35]$.

Regarding particle shape, we observed that spherical and discoidal particles outperformrod-like particles, in agreement with a recent computational study in which theprobability of a particleto 
exhibit a "waterfall motion" depended greatly on its shape[31].In particular, in the present study, discoidal and spherical particles exhibited a higher frequency of collision with RBCs than did rodlike particles, due to the orientation of the latter along the capillary axis.Since discs exhibited a flipping and tumbling motion around their longitudinal axis, oriented as the flow direction, they hada higher probability to interact with RBCs. On the contrary, rod-like particles performed a revolution around their minor axis (i.e., the axis perpendicular to the longitudinal axis), during their flow along the streamlines, and were thus less likely to collide with RBCs[31].

\section{Conclusions}

This work provides a quantitative analysis of the influence of RBCs on the flow and margination dynamics of micron-sized drug carriersin vitro and by intravital microscopy in vivo. Our in vitro studies mimicthe mechanisms that regulate the transport of injectable carriers in the microcirculation, elucidating the effect of particle size and shape on particle transport towards the vessel vasculature in micro-capillary flow. In fact, the influence of RBCs on the transport, distribution and accumulation of drug carriers in micro-capillaries, as a function of their size and shape, should be taken into consideration when designing and engineering systemically administered drug delivery carriers. Our resultsshowthat margination, which is almost absent when particles are suspended in a cell-free medium, is drastically enhanced by RBCs and that this effect is size- and shape-dependent. We observed that 3- $\mu \mathrm{m}$ spheres marginate more efficientlythan 1- $\mu \mathrm{m}$ spheres, in accordance with previous reports in the literature $[9,71]$, and confirming a numerical study that shows that the optimal dimension for marginationis 2-3 $\mu \mathrm{m}[35]$.

Regarding the effect of shape on particle transport, discoidal and spherical particles performed best in terms of margination dynamics, makingflat, discoidal particles optimally suited to increase wall adhesion (akin to platelets), due to their high contact surface area [35]. Moreover, as reported in a recent study on biodistribution and tumoritropic accumulation, discoidal particles show high accumulation efficiency in tumor also in absence of a specific targeting ligand [53].

In conclusion, an increase in the margination dynamics and in the probability of stable adhesion of injectable drug delivery systems to the vessel wall can be achieved by engineering carriers with a platelet-like shape and with large, flat exposed surface areas. Our microfluidics approach provides a methodology to single out the effect of margination of drug carriers and can thus be relevant for the design of novel therapeutic systems for systemic administration.

\section{Acknowledgments}

Funding from the Italian Ministry of University and Research (PRIN Program 2010-2011, Project No. 20109PLMH2), and from the Regione Campania (MICROEMA Project, 220 APQRT02 2008) is acknowledged.This work has been also supported by POR CAMPANIA FSE 2007-2013 Project DIAINTECH, Italy (to F.S.) and by Grant PON01_02589 (MICROMAP) - 2012 from the Ministry of University and Research, Italy (to F.S.)

This study is related to the activity of the European network action COST MP1106 "Smart and green interfaces - from single bubbles and drops to industrial, environmental, and biomedical applications."The authors acknowledge Dr. YeonJu Lee for figure design. The authors gratefully acknowledge funding support from the following sources: Department of Defense grants 
W81XWH-09-1-0212 and W81XWH-12-1-0414, National Institute of Health grants U54CA143837 and U54CA151668, the CPRIT grant RP121071 from the State of Texas, and the Ernest Cockrell Jr. Distinguished Endowed Chair.This study was also supported by the Brown Foundation (Project ID: 18130011) and by the Cullen Trust for Health Care Foundation (Project ID: 18130014). We thank Jean Ann Gilder (Scientific Communication srl, Naples, Italy) for text editing.

\section{References}

[1] U. Prabhakar, H. Maeda, R. K. Jain, E. M. Sevick-Muraca, W. Zamboni, O. C. Farokhzad, S. T. Barry, A. Gabizon, P. Grodzinski, and D. C. Blakey, "Challenges and Key Considerations of the Enhanced Permeability and Retention Effect for Nanomedicine Drug Delivery in Oncology," Cancer Research, vol. 73, no. 8, pp. 2412-2417, April 15, 2013, 2013.

[2] S. T. Proulx, P. Luciani, L. C. Dieterich, S. Karaman, J.-C. Leroux, and M. Detmar, "Expansion of the lymphatic vasculature in cancer and inflammation: New opportunities for in vivo imaging and drug delivery," Journal of Controlled Release, vol. 172, no. 2, pp. 550557, 12/10/, 2013.

[3] P. Yousefpour, and A. Chilkoti, "Co-opting biology to deliver drugs," Biotechnol Bioeng, vol. 111, no. 9, pp. 1699-716, Sep, 2014.

[4] R. A. Petros, and J. M. DeSimone, "Strategies in the design of nanoparticles for therapeutic applications," Nat Rev Drug Discov, vol. 9, no. 8, pp. 615-627, 08//print, 2010.

[5] V. V. Ranade, and J. B. Cannon, Drug delivery systems: CRC press, 2011.

[6] M. Ferrari, "Cancer nanotechnology: opportunities and challenges," Nat Rev Cancer, 3, pp. 161-71, England, 2005.

[7] J. W. Nichols, and Y. H. Bae, "Odyssey of a cancer nanoparticle: From injection site to site of action," Nano Today, vol. 7, no. 6, pp. 606-618, 12//, 2012.

[8] F. Gentile, A. Curcio, C. Indolfi, M. Ferrari, and P. Decuzzi, "The margination propensity of spherical particles for vascular targeting in the microcirculation," Journal of nanobiotechnology, vol. 6, pp. 9-9, 2008 Aug, 2008.

[9] K. Namdee, A. J. Thompson, P. Charoenphol, and O. Eniola-Adefeso, "Margination Propensity of Vascular-Targeted Spheres from Blood Flow in a Microfluidic Model of Human Microvessels," Langmuir, Feb 8, 2013.

[10] R. Toy, P. M. Peiris, K. B. Ghaghada, and E. Karathanasis, "Shaping cancer nanomedicine: the effect of particle shape on the in vivo journey of nanoparticles," Nanomedicine (Lond), vol. 9, no. 1, pp. 121-34, Jan, 2014.

[11] J. Tan, S. Shah, A. Thomas, H. D. Ou-Yang, and Y. Liu, "The influence of size, shape and vessel geometry on nanoparticle distribution," Microfluidics and Nanofluidics, vol. 14, no. 1-2, pp. 77-87, 2013/01/01, 2013.

[12] E. Carboni, K. Tschudi, J. Nam, X. Lu, and A. W. Ma, "Particle margination and its implications on intravenous anticancer drug delivery," AAPS PharmSciTech, vol. 15, no. 3, pp. 762-71, Jun, 2014

[13] N. Doshi, B. Prabhakarpandian, A. Rea-Ramsey, K. Pant, S. Sundaram, and S. Mitragotri, "Flow and adhesion of drug carriers in blood vessels depend on their shape: a study using model synthetic microvascular networks," Journal of Controlled Release, vol. 146, no. 2, pp. 196-200, 2010.

[14] G. Tomaiuolo, "Biomechanical properties of red blood cells in health and disease towards microfluidics," Biomicrofluidics, vol. 8, no. 5, pp. -, 2014.

[15] S. Guido, and G. Tomaiuolo, "Microconfined flow behavior of red blood cells in vitro," $C$. R. Physique, vol. 10, pp. 751-763, 2009. 
[16] G. Tomaiuolo, V. Preziosi, M. Simeone, S. Guido, R. Ciancia, V. Martinelli, C. Rinaldi, and B. Rotoli, "A methodology to study the deformability of red blood cells flowing in microcapillaries in vitro.," Ann Ist Super Sanita, vol. 43, no. 2, pp. 186-92, 2007.

[17] G. Tomaiuolo, M. Simeone, V. Martinelli, B. Rotoli, and S. Guido, "Red blood cell deformability in microconfined shear flow," Soft Matter, vol. 5, pp. 3736-3740, 2009.

[18] G. Tomaiuolo, M. Barra, V. Preziosi, A. Cassinese, B. Rotoli, and S. Guido, "Microfluidics analysis of red blood cell membrane viscoelasticity.," Lab Chip, vol. 11, no. 3, pp. 449-54, Feb, 2011.

[19] G. Tomaiuolo, and S. Guido, "Start-up shape dynamics of red blood cells in microcapillary flow," Microvasc Res, vol. 82, no. 1, pp. 35-41, Jul, 2011.

[20] G. Tomaiuolo, D. Rossi, S. Caserta, M. Cesarelli, and S. Guido, "Comparison of two flowbased imaging methods to measure individual red blood cell area and volume," Cytom Part $A$, vol. 81, no. 12, 2012-Dec, 2012.

[21] G. Tomaiuolo, L. Lanotte, G. Ghigliotti, C. Misbah, and S. Guido, "Red blood cell clustering in Poiseuille microcapillary flow," Physics of Fluids, vol. 24, no. 5, pp. 051903-8, 05/00/, 2012.

[22] L. Lanotte, G. Tomaiuolo, C. Misbah, L. Bureau, and S. Guido, "Red blood cell dynamics in polymer brush-coated microcapillaries: A model of endothelial glycocalyx in vitro," Biomicrofluidics, vol. 8, no. 1, pp. -, 2014.

[23] J. Tan, A. Thomas, and Y. Liu, "Influence of red blood cells on nanoparticle targeted delivery in microcirculation," Soft Matter, vol. 8, no. 6, pp. 1934-1946, 2012, 2012.

[24] U. Nobis, A. Pries, G. Cokelet, and P. Gaehtgens, "Radial distribution of white cells during blood flow in small tubes," Microvascular research, vol. 29, no. 3, pp. 295-304, 1985.

[25] P. Aarts, S. A. T. Vandenbroek, G. W. Prins, G. D. C. Kuiken, J. J. Sixma, and R. M. Heethaar, "Blood-platelets are concentrated near the wall and red blood-cells, in the center in flowing blood," Arteriosclerosis, vol. 8, no. 6, pp. 819-824, Nov-Dec, 1988.

[26] C. J. Yeh, and E. C. Eckstein, "Transient lateral transport of platelet-sized particles in flowing blood suspensions," Biophysical Journal, vol. 66, no. 5, pp. 1706-1716, May, 1994.

[27] R. Zhao, M. V. Kameneva, and J. F. Antaki, "Investigation of platelet margination phenomena at elevated shear stress," Biorheology, vol. 44, no. 3, pp. 161-177, 2007, 2007.

[28] H. Zhao, E. S. G. Shaqfeh, and V. Narsimhan, "Shear-induced particle migration and margination in a cellular suspension," Physics of Fluids, vol. 24, no. 1, pp. 011902-21, 2012.

[29] T. M. Geislinger, and T. Franke, "Hydrodynamic lift of vesicles and red blood cells in flow - from Fåhræus \&amp; Lindqvist to microfluidic cell sorting," Advances in Colloid and Interface Science, no. 0.

[30] M. Saadatmand, T. Ishikawa, N. Matsuki, M. J. Abdekhodaie, Y. Imai, H. Ueno, and T. Yamaguchi, "Fluid particle diffusion through high-hematocrit blood flow within a capillary tube," Journal of Biomechanics, vol. 44, no. 1, pp. 170-175, Jan 4, 2011.

[31] K. Vahidkhah, and P. Bagchi, "Microparticle shape effects on margination, near-wall dynamics and adhesion in a three-dimensional simulation of red blood cell suspension," Soft matter, 2015.

[32] C. Xu, and D. M. Wootton, "Platelet near-wall excess in porcine whole blood in artery-sized tubes under steady and pulsatile flow conditions," Biorheology, vol. 41, no. 2, pp. 113-125, 2004.

[33] T.-R. Lee, M. Choi, A. M. Kopacz, S.-H. Yun, W. K. Liu, and P. Decuzzi, "On the nearwall accumulation of injectable particles in the microcirculation: smaller is not better," Scientific Reports, vol. 3, Jun 26, 2013.

[34] P. Decuzzi, B. Godin, T. Tanaka, S. Y. Lee, C. Chiappini, X. Liu, and M. Ferrari, "Size and shape effects in the biodistribution of intravascularly injected particles," Journal of Controlled Release, vol. 141, no. 3, pp. 320-327, Feb 15, 2010. 
[35] K. Mueller, D. A. Fedosov, and G. Gompper, "Margination of micro- and nano-particles in blood flow and its effect on drug delivery," Scientific Reports, vol. 4, May 2, 2014.

[36] S.-Y. Lee, M. Ferrari, and P. Decuzzi, "Shaping nano-/micro-particles for enhanced vascular interaction in laminar flows," Nanotechnology, vol. 20, no. 49, Dec 9, 2009.

[37] P. Decuzzi, and M. Ferrari, "The adhesive strength of non-spherical particles mediated by specific interactions," Biomaterials, vol. 27, no. 30, pp. 5307-5314, Oct, 2006.

[38] F. Gentile, C. Chiappini, D. Fine, R. C. Bhavane, M. S. Peluccio, M. M.-C. Cheng, X. Liu, M. Ferrari, and P. Decuzzi, "The effect of shape on the margination dynamics of nonneutrally buoyant particles in two-dimensional shear flows," Journal of Biomechanics, vol. 41, no. 10, pp. 2312-2318, Jul 19, 2008.

[39] R. Toy, E. Hayden, C. Shoup, H. Baskaran, and E. Karathanasis, "The effects of particle size, density and shape on margination of nanoparticles in microcirculation," Nanotechnology, vol. 22, no. 11, pp. 115101, 2011.

[40] A. J. Thompson, E. M. Mastria, and O. Eniola-Adefeso, "The margination propensity of ellipsoidal micro/nanoparticles to the endothelium in human blood flow," Biomaterials, 23, pp. 5863-71, Netherlands: 2013 Elsevier Ltd, 2013.

[41] M. Toner, and D. Irimia, "Blood-on-a-chip.," Annu Rev Biomed Eng, vol. 7, pp. 77-103, 2005.

[42] G. M. Whitesides, "The origins and the future of microfluidics," Nature, vol. 442, no. 7101, Jul 27, 2006.

[43] X. Jiang, Q. Xu, S. Dertinger, A. Stroock, T. Fu, and G. Whitesides, "A general method for patterning gradients of biomolecules on surfaces using microfluidic networks," Anal Chem, vol. 77, no. 8, pp. 2338-47, Apr, 2005.

[44] B. S. Ding, T. Dziubla, V. V. Shuvaev, S. Muro, and V. R. Muzykantov, "Advanced drug delivery systems that target the vascular endothelium," Mol Interv, 2, pp. 98-112, United States, 2006.

[45] E. Blanco, A. Sangai T Fau - Hsiao, S. Hsiao A Fau - Ferrati, L. Ferrati S Fau - Bai, X. Bai L Fau - Liu, F. Liu X Fau - Meric-Bernstam, M. Meric-Bernstam F Fau - Ferrari, and M. Ferrari, "Multistage delivery of chemotherapeutic nanoparticles for breast cancer treatment," no. 1872-7980 (Electronic), 20130531 DCOM- 20131210.

[46] D. J. Savage, X. Liu, S. A. Curley, M. Ferrari, and R. E. Serda, "Porous silicon advances in drug delivery and immunotherapy," Curr Opin Pharmacol, vol. 13, no. 5, pp. 834-41, Oct, 2013.

[47] F. Taraballi, S. Minardi, B. Corradetti, I. K. Yazdi, M. A. Balliano, J. L. Van Eps, M. Allegri, and E. Tasciotti, "Potential Avoidance of Adverse Analgesic Effects Using a Biologically "Smart" Hydrogel Capable of Controlled Bupivacaine Release," Journal of Pharmaceutical Sciences, vol. 103, no. 11, pp. 3724-3732, Nov, 2014.

[48] J. M. Lu, X. Wang, C. Marin-Muller, H. Wang, P. H. Lin, Q. Yao, and C. Chen, "Current advances in research and clinical applications of PLGA-based nanotechnology," Expert Rev Mol Diagn, vol. 9, no. 4, pp. 325-41, May, 2009.

[49] F. Danhier, E. Ansorena, J. M. Silva, R. Coco, A. Le Breton, and V. Préat, "PLGA-based nanoparticles: An overview of biomedical applications," Journal of Controlled Release, vol. 161, no. 2, pp. 505-522, 7/20/, 2012.

[50] S. P. Egusquiaguirre, M. Igartua, R. M. Hernandez, and J. L. Pedraz, "Nanoparticle delivery systems for cancer therapy: advances in clinical and preclinical research," Clin Transl Oncol, 2, pp. 83-93, Italy, 2012.

[51] C. Chiappini, E. Tasciotti, J. R. Fakhoury, D. Fine, L. Pullan, Y. C. Wang, L. Fu, X. Liu, and M. Ferrari, "Tailored porous silicon microparticles: fabrication and properties," Chemphyschem, vol. 11, no. 5, pp. 1029-35, Apr 6, 2010.

[52] E. Tasciotti, X. Liu, R. Bhavane, K. Plant, A. D. Leonard, B. K. Price, M. M. Cheng, P. Decuzzi, J. M. Tour, F. Robertson, and M. Ferrari, "Mesoporous silicon particles as a 
multistage delivery system for imaging and therapeutic applications," Nat Nanotechnol, vol. 3, no. 3, pp. 151-7, Mar, 2008.

[53] A. L. van de Ven, P. Kim, O. Haley, J. R. Fakhoury, G. Adriani, J. Schmulen, P. Moloney, F. Hussain, M. Ferrari, X. Liu, S. H. Yun, and P. Decuzzi, "Rapid tumoritropic accumulation of systemically injected plateloid particles and their biodistribution," $J$ Control Release, vol. 158, no. 1, pp. 148-55, Feb 28, 2012.

[54] J. O. Martinez, A. Parodi, X. Liu, M. G. Kolonin, M. Ferrari, and E. Tasciotti, "Evaluation of cell function upon nanovector internalization," Small, vol. 9, no. 9-10, pp. 1696-702, May 27, 2013.

[55] Y.-c. Fung, Biomechanics: circulation: Springer, 1997.

[56] K. Albrecht, P. Gaehtgens, A. Pries, and M. Heuser, "The Fahraeus effect in narrow capillaries (i.d. 3.3 to 11.0 micron)," Microvasc Res, vol. 18, no. 1, pp. 33-47, Jul, 1979.

[57] R. L. Whitmore, "The flow behaviour of blood in the circulation," Nature, vol. 215, no. 5097, pp. 123-6, 1967-Jul-8, 1967.

[58] M. Smith, D. Long, E. Damiano, and K. Ley, "Near-wall micro-PIV reveals a hydrodynamically relevant endothelial surface layer in venules in vivo.," Biophys $J$, vol. 85, no. 1, pp. 637-45, Jul, 2003.

[59] A. S. Popel, and P. C. Johnson, "Microcirculation and hemorheology," Annual Review of Fluid Mechanics, vol. 37, no. 1, pp. 43-69, 2005.

[60] R. B. Bird, W. E. Stewart, and E. N. Lightfoot, Transport phenomena: John Wiley \& Sons, 2007.

[61] C. Misbah, "Vesicles, capsules and red blood cells under flow," Journal of Physics: Conference Series, vol. 392, no. 1, pp. 012005, 2012.

[62] A. Pommella, S. Caserta, and S. Guido, "Dynamic flow behaviour of surfactant vesicles under shear flow: role of a multilamellar microstructure," Soft Matter, vol. 9, no. 31, pp. 7545-7552, 2013.

[63] P. Bagchi, and R. M. Kalluri, "Dynamics of nonspherical capsules in shear flow," Physical Review E, vol. 80, no. 1, Jul, 2009.

[64] J. Walter, A. V. Salsac, and D. Barthes-Biesel, "Ellipsoidal capsules in simple shear flow: prolate versus oblate initial shapes," Journal of Fluid Mechanics, vol. 676, pp. 318-347, Jun, 2011.

[65] G. Segre, and A. Silberberg, "Radial Particle Displacements in Poiseuille Flow of Suspensions," Nature, vol. 189, no. 4760, pp. 209-210, 01/21/print, 1961.

[66] K. Vahidkhah, Scott L. Diamond, and P. Bagchi, "Platelet Dynamics in Three-Dimensional Simulation of Whole Blood," Biophysical Journal, vol. 106, no. 11, pp. 2529-2540, 6/3/, 2014.

[67] D. A. Fedosov, B. Caswell, A. S. Popel, and G. E. Karniadakis, "Blood flow and cell-free layer in microvessels," Microcirculation, vol. 17, no. 8, pp. 615-28, Nov, 2010.

[68] B. Woldhuis, G. J. Tangelder, D. W. Slaaf, and R. S. Reneman, "Concentration profile of blood-platelets differs in arterioles and venules," American Journal of Physiology, vol. 262, no. 4, pp. H1217-H1223, Apr, 1992.

[69] L. M. Crowl, and A. L. Fogelson, "Computational model of whole blood exhibiting lateral platelet motion induced by red blood cells," International Journal for Numerical Methods in Biomedical Engineering, vol. 26, no. 3-4, pp. 471-487, Mar-Apr, 2010.

[70] G. Adriani, M. D. de Tullio, M. Ferrari, F. Hussain, G. Pascazio, X. Liu, and P. Decuzzi, "The preferential targeting of the diseased microvasculature by disk-like particles," Biomaterials, vol. 33, no. 22, pp. 5504-13, Aug, 2012.

[71] P. Charoenphol, P. J. Onyskiw, M. Carrasco-Teja, and O. Eniola-Adefeso, "Particle-cell dynamics in human blood flow: Implications for vascular-targeted drug delivery," Journal of Biomechanics, vol. 45, no. 16, pp. 2822-2828, Nov 15, 2012. 

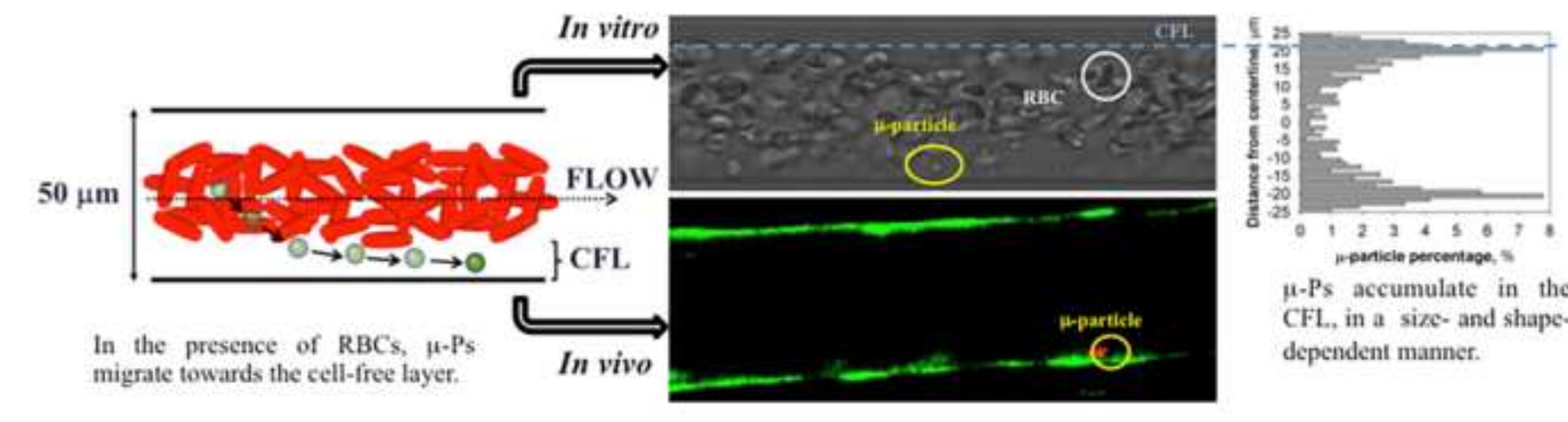

$\mu$-Ps accumulate in the CFL, in a size- and shapedependent manner.

In the presence of $\mathrm{RBCs}$, $\mu-\mathrm{P}$

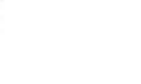

